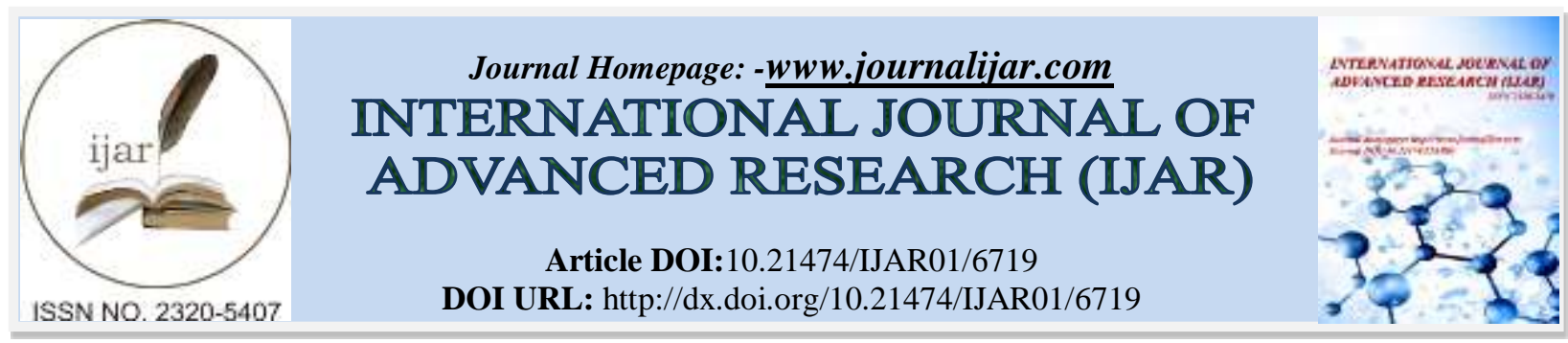

RESEARCH ARTICLE

\title{
INNOVATIVE TECHNIQUE FOR RESTORATION OF OPTICAL COHERENCE TOMOGRAPHY IMAGES AND COMPARISON WITH BM3D.
}

\author{
Vinod Kumar Netad. \\ Department of Electrical Engineering, IIT Hyderabad, India.
}

\section{Manuscript Info}

Manuscript History

Received: 10 January 2018

Final Accepted: 12 February 2018

Published: March 2018

Keywords:-

Optical Coherence Tomography (OCT), Choroid, Retinal Pigment Epithelium (RPE), Otsu's method, Discrete wavelet transform (DWT), Inverse discrete Wavelet Transform (IDWT)

\begin{abstract}
The automated processing of Optical Coherence tomography images has main difficulty of presence of noise which varies over the entire image. We first segment the OCT image into two regions, one containing different layers of eye and the other which contains only noise. The similar rows which contain only noise are considered to model the noise and then we find the local threshold value in adaptive manner. We were formulating a novel algorithm for OCT image restoration which uses a threshold derived from mean and variance of discrete wavelet coefficients in different sub bands of noisy image (segment of OCT image containing only noise). The found threshold will be different for the two segments of the image and all their sub bands. Thus this algorithm won't smooth the entire image and preserves the edge information and removes the noise. We compared our results with one of the most efficient technique BM3D.
\end{abstract}

Copy Right, IJAR, 2018,. All rights reserved.

\section{Introduction:-}

The OCT image is captured by interference of the light coming by the reflection of light from different layers of the eye. There are different layers of eye like Retinal Pigment Epithelium (RPE), Choroid; Sclera etc. are present behind retina which is captured in OCT images. Different parts of the layers have different reflective properties. So the information is captured as a gray level image. But because of the addition of the noise and the poor resolution of low cost OCT imaging machines, the information may not be viewed properly by the clinician. For restoring the image we need the information about the noise. In most of the cases the noise added to the OCT images is found experimentally to be Gaussian in nature with small tails. OCT image can mainly be viewed as a two level image, brighter pixels which are formed by reflective regions and darker pixels are not reflective like vessels in choroid. The noise in darker regions is added like a Gaussian variable added to a constant mean value in a row. In brighter region, there is some information with added noise. By using this hypothesis that in darker regions contain less information, more noise we restore the image in adaptive manner.

The Wavelet transform of the image is the representation of the image with the help of orthonormal basis functions. It contains different sub bands which contains different set of frequencies. Noise normally affects a set of frequencies, it will have a frequency spectrum which can be approximated by seeing the sub bands of the Wavelet decomposed noisy image. Now by knowing the mostly effected sub band we will know the sub band of the original segment which contains noise. Then we will estimate the effected sub band coefficients which correspond to noise, found by local threshold (soft) for the Wavelet transform from local neighborhood. We were doing suppression of wavelet coefficients belonging to noise adaptive by performing local operations on DWT of the OCT image segment 
with layers motivated by [1]. The darker region contains mostly noise, so the large coefficients belong to noise. And the brighter region contains more information than noise, so large coefficients correspond to edge information and smaller coefficients correspond to noise. By using this we will modify the coefficients, then find the inverse of the wavelet transform to find approximate restored image.

Most efficient state of art technique is BM3D. It uses the local sparse representation in transform domain, then form 3D image by grouping similar patches, and then do filtering to remove noise, and then apply inverse 3D transform to restore the image. But the application of this technique is not preserving much edge information, it has more smoothing effect. So we were formulating an algorithm for restoration of OCT images, which preserves more edge information.

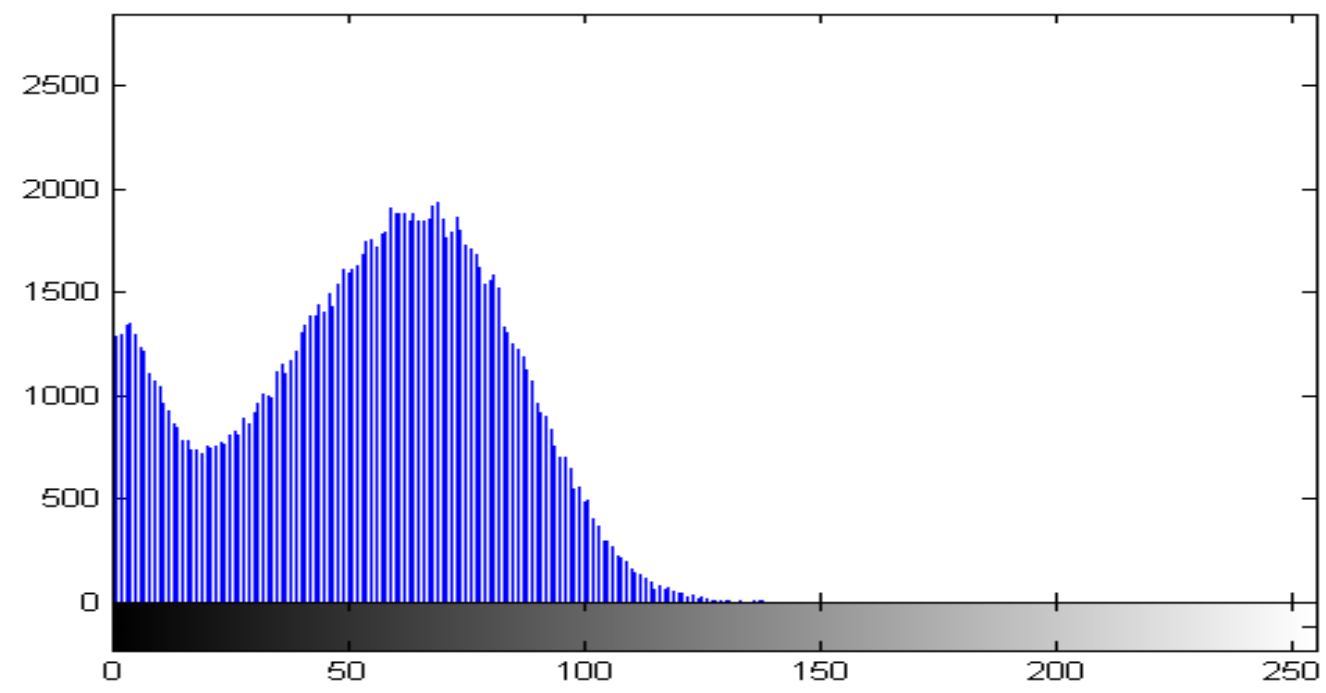

Fig 1:-Noise Histogram

\section{Proposed Algorithm:-}

\section{Segmenting OCT image into noisy image and information image:}

By using the experimental result that the histograms of the noisy regions look like Gaussian distributions with shifted means as in Fig.1. The mean intensities of the rows in the OCT image will decrease when we traverse from choroid to the end of the image. Because of the attenuation of the light in the eye, even in the choroid region the mean intensity of the rows decreases. From the experiments we have known that the mean intensity of the row in the noisy region has a very high probability of having very low mean intensity than the mean intensity of the rows in the segment containing information of layers of eye. With the help of these two results we will divide the OCT image into segments noisy region and layers of eye. 


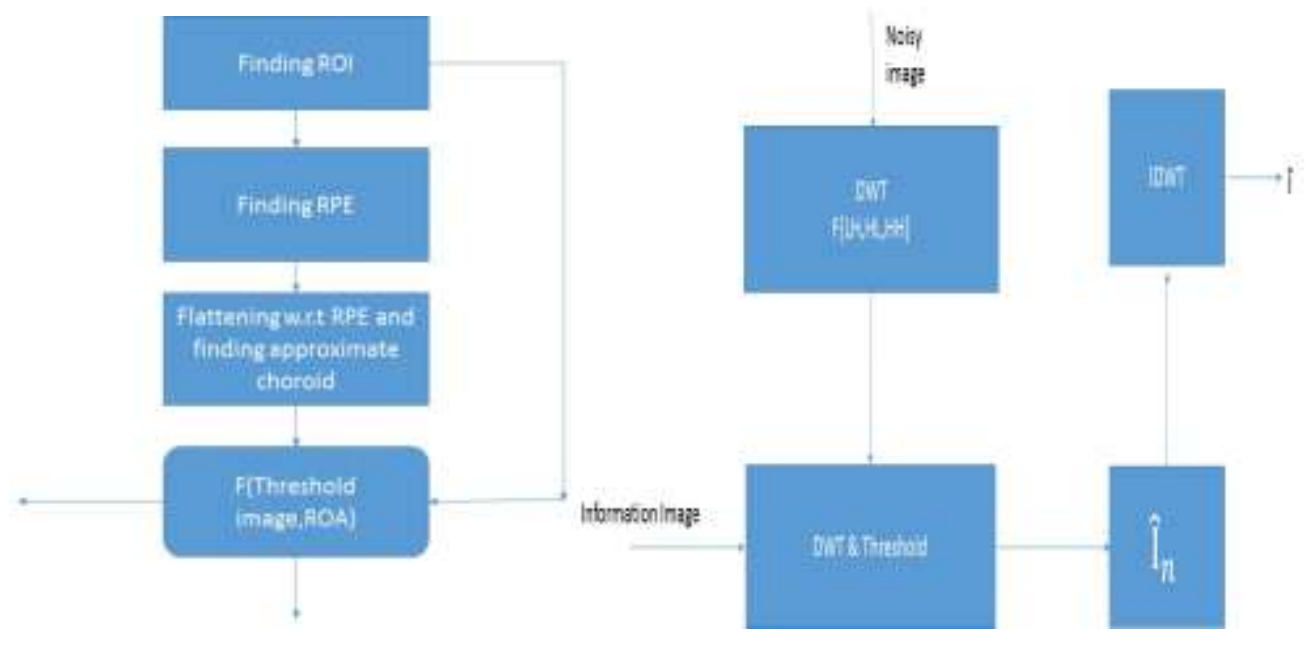

Fig 2:- Segmentation

Fig 3:- Algorithm

We will find the Retinal Pigment Epithelium (RPE) with help of intensity and gradient properties of it as in [2]. Then taking RPE as reference we will flatten the OCT image. The local threshold uses Otsu's method [3] on the flattened version of OCT in local overlapping windows to find local thresholds. By using this binary image we will find the dark region which contains mostly vessels. These vessels won't reflect any light, so we were using the hypothesis that the darker region even in this segment contains mostly noise. And the information content is very low. And the brighter region contains most information and noise. So we will restore them differently.

\section{Finding soft threshold:-}

We will first decide the level of decomposition. The restoration of the image of size $N_{1} \times N_{2}$ is very much dependent on the choice of the level of decomposition $(\mathrm{m})$. As the level of wavelet decomposition increases the low range of frequency sub band increases, and from the observations we have known that the noise added to the noise is of speckle type, which occurs as sudden fluctuations and information is present in low frequencies and at edges there are some high frequencies which possess information as well, which can be retained by observing local neighborhood. So we experimentally used 3level decomposition which gives good results. We first should resize the image to be of height and width which are multiples of $M$, where $M$ is power of 2 of order chosen level (m). In our experiments the images are resized to multiple of 8 , are two level discrete wavelet transform and its inverse.

The two level discrete wavelet transform produces 4 sub bands $\{\mathrm{LL}, \mathrm{LH}, \mathrm{HL}, \mathrm{HH}$ \}. We assumed that LH, HL, HH sub bands only contain noise and LL contains only information. Depending on the level of decomposition we will keep on doing two level wavelet transform, so there will be these sub bands at various scales $N_{1} / 2^{K} \times N_{2} / 2^{K}$, where scale decreases with level. By the experiments we have chosen threshold to be soft.

\section{Finding noise parameters mean and variance:-}

We assumed that noise is additive in nature. By observing the fig 1, we can see that noise image histogram; distribution of levels follows a particular distribution. So we can approximate the noise from a Gaussian model. We should find the mean $(\eta)$ and variance $\left(\sigma^{2}\right)$ of the noise added from that. Now at each level of decomposition of noisy image $\left(\eta=\eta+\mathrm{N}\left(0, \sigma^{2}\right)\right)$ we will find the mean of the wavelet coefficients, and the standard deviation, mean added with a multiple of standard deviation is taken as soft threshold value $\left(T_{n}\right)$ motivated by 4 . The noisy coefficients in noisy image will have higher value and in information image will be smaller. So accordingly we will replace the noisy coefficients with wiener filtered (with found $n, s)$ sub band value. This retains high frequency edges belonging to information because of more number of high values in neighborhood. This removes Gaussian noise.

$$
\begin{gathered}
\mathrm{n}=\sum \eta(\mathrm{i}, \mathrm{j}) \\
\sigma=\left(\sum \eta(i, j)^{2}-n^{2}\right)^{1 / 2}
\end{gathered}
$$

1. Multi-level decomposition

2. Wavelet transform, Finding noise mean and variance and wavelet threshold for various high frequency sub bands

3. Threshold wavelet coefficients noisy segment 
4. Threshold information segment

5. Inverse wavelet transform to find approximate restored image

$$
\begin{aligned}
& \hat{I}=\left(I_{n}, T_{n}\right) \\
& \widehat{I}=W^{-1} * \hat{I}_{n}
\end{aligned}
$$

We will use various discrete wavelet transforms (DWT) with different analysis filters like Haar [5] , Daubechies [6] etc. with different levels of decomposition and find the dominant sub band of the noisy image and we will know which is the sub band effected by noise, and distribution of noise. We will do local threshold of the OCT image segment with layers of the eye.

\section{Result:-}

We have used 30 very noisy Standard Definition OCT images (SD-OCT) provided by LV Prasad Eye Institute (LVPEI) as dataset to test our algorithm. We have used HAAR wavelet as wavelet basis and 3 level decomposition from experiments to test our algorithm. And we will test the restored image in the algorithm used in [2] and compare the final results. And compare the binary images after and before restoration of the images. We have used MATLAB as the tool to test the algorithm. We have taken wiener filter from MATLAB with $3 \times 3$ windows to approximate the noisy coefficients.

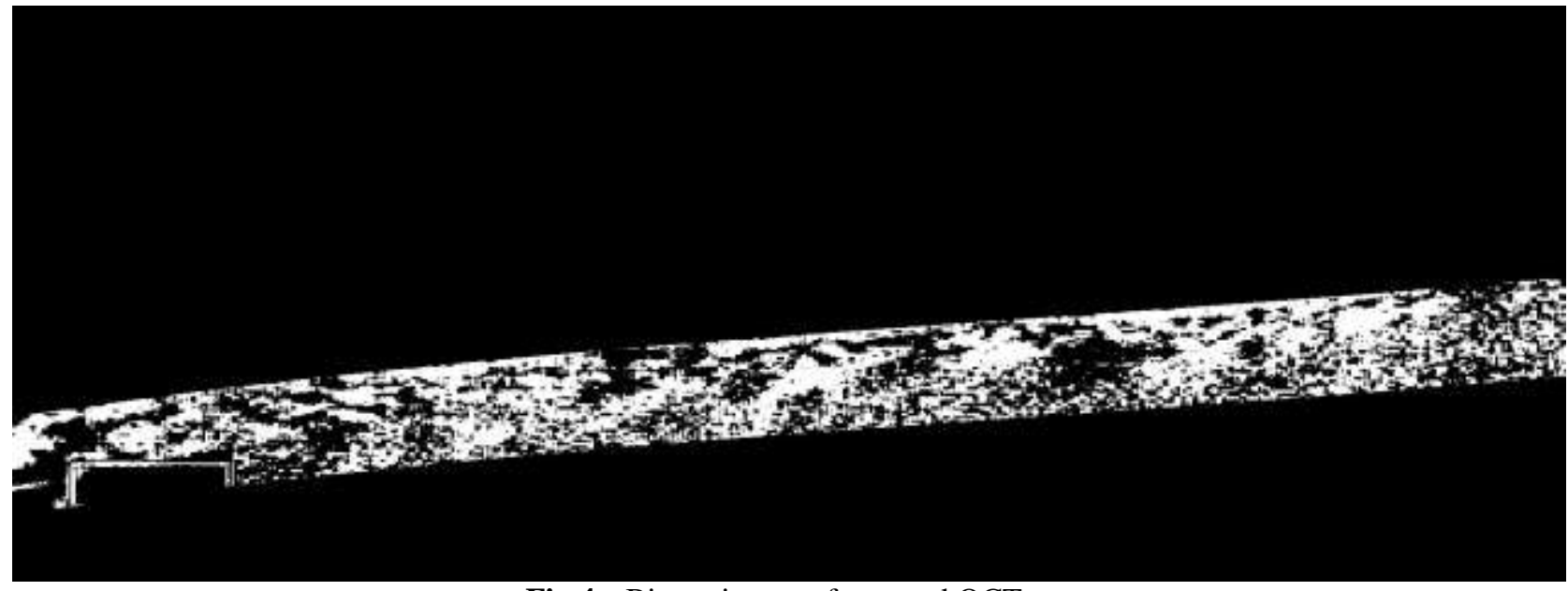

Fig 4:- Binary image of restored OCT

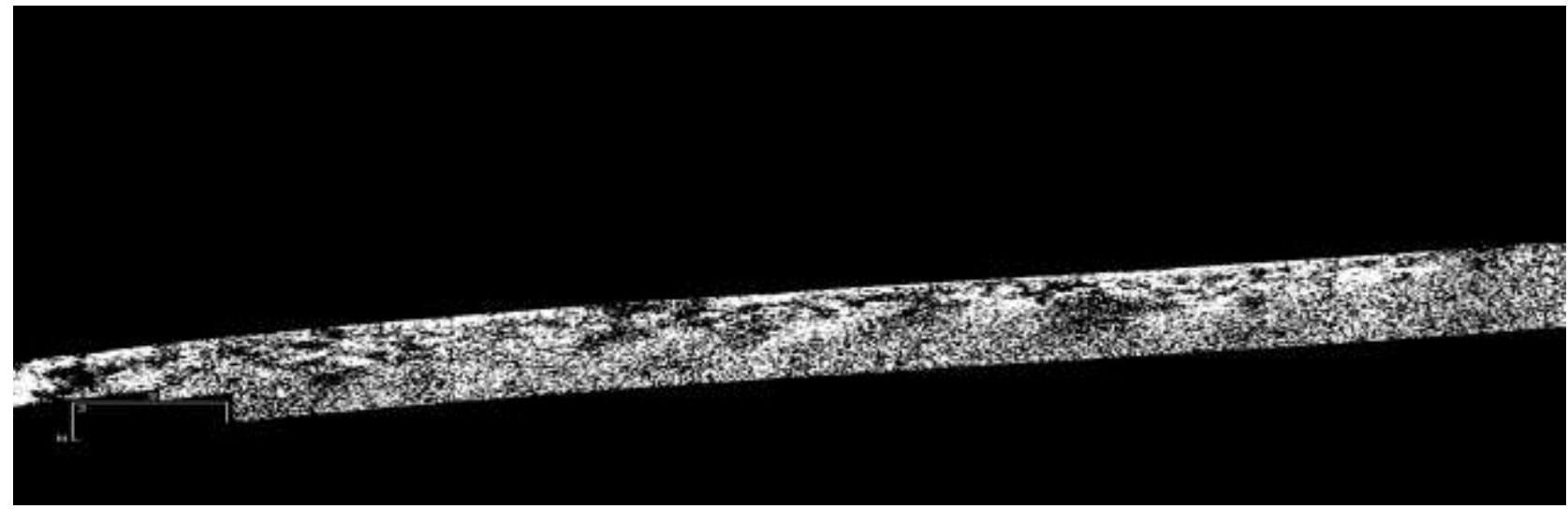

Fig 5:- Binary image of original OCT 


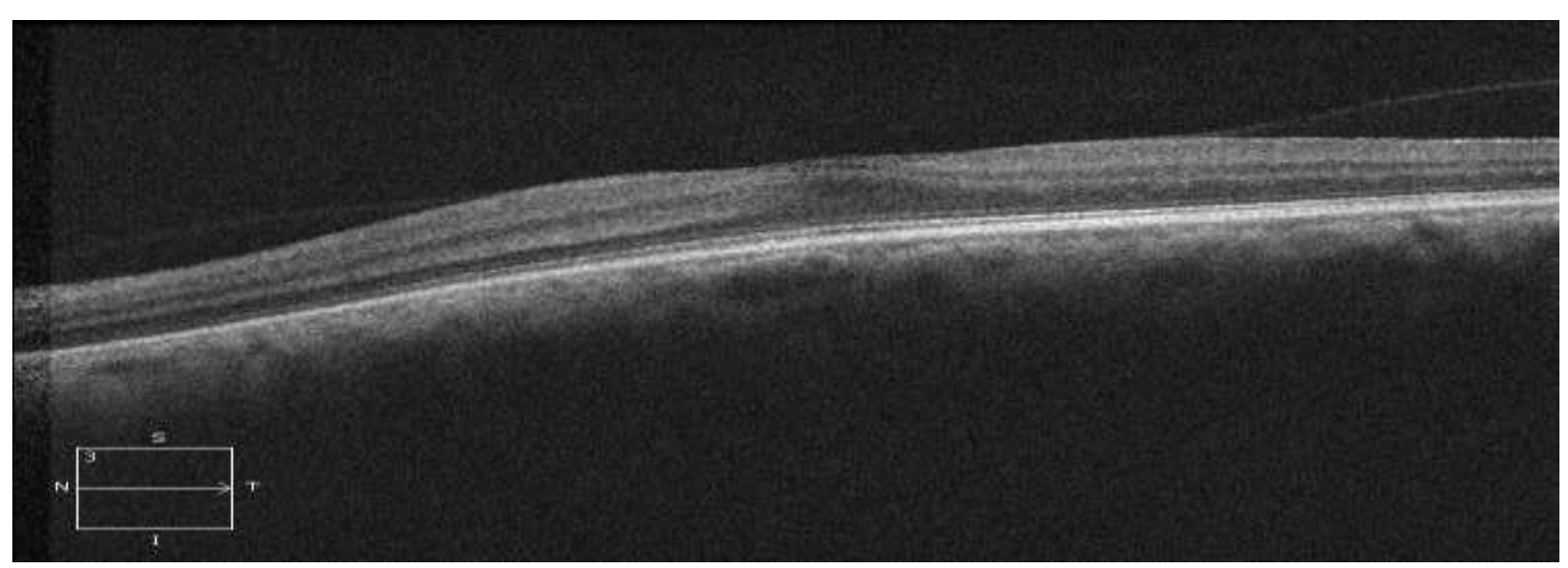

Fig.6:-Low SNR OCT image

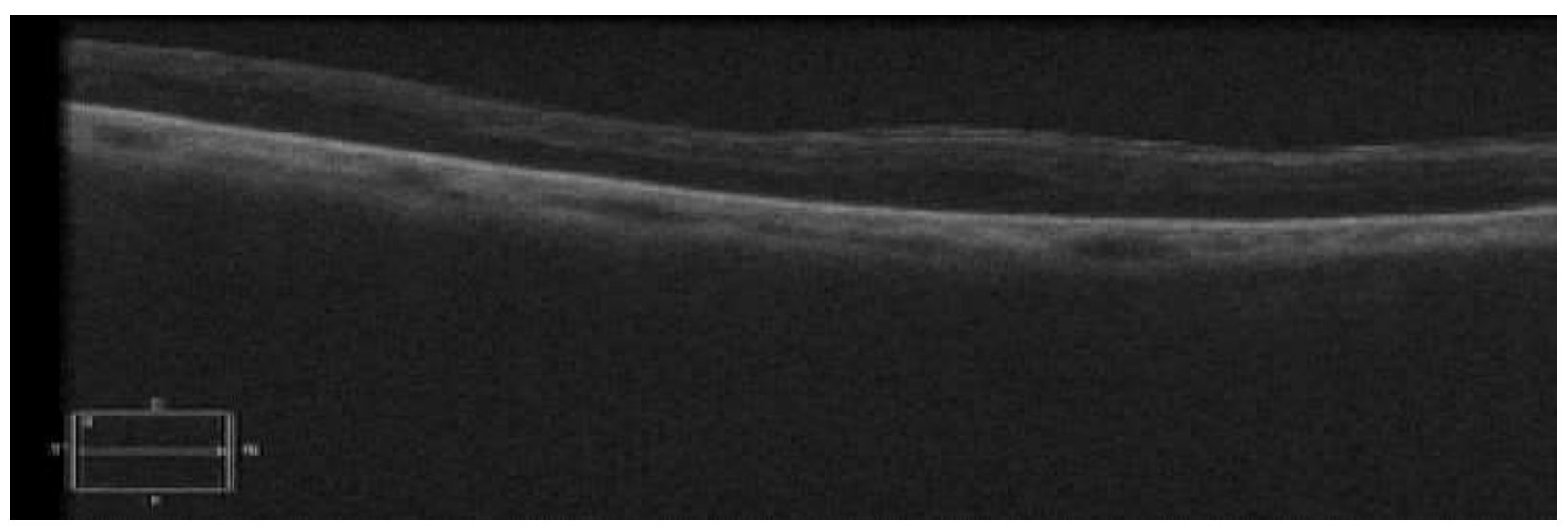

Fig.7:- Low SNR Denoised OCT image

The main problem with present BM3D technique is that, its removes noise efficiently but the smoothing effect caused by it more. The main information as discussed is stored in the form of gradient information because of which we can perceive different types of layers present. As discussed there are only two types of material, reflective and attenuating (blood vessels). So when we find binary image by doing local threshold, we will separate these two kinds regions. Because of the smoothing the binary image formed is connected such that, the darker regions or brighter regions in small neighborhood are merged. From our restoring technique, see figures 5, 4 we have shown that our algorithm preserves edge information while suppressing noise.

Another disadvantage we were considering was the poorly visible images as in Fig.6, which are very difficult for the clinician to take a decision. Most of the present algorithms are not adaptive, even though they consider local neighborhood because of the application of same soft threshold over darker as well as brighter regions, the edge information preserved will be very less. Our algorithm guarantees more edge preserving capability as shown in Fig.7.

We compared the restored HD-OCT image by our algorithm (see Fig.8 and Fig.9 9 ) and, state of art BM3D. We use HAAR basis with 3 level decomposition, and soft threshold as mean of the sub band image. 


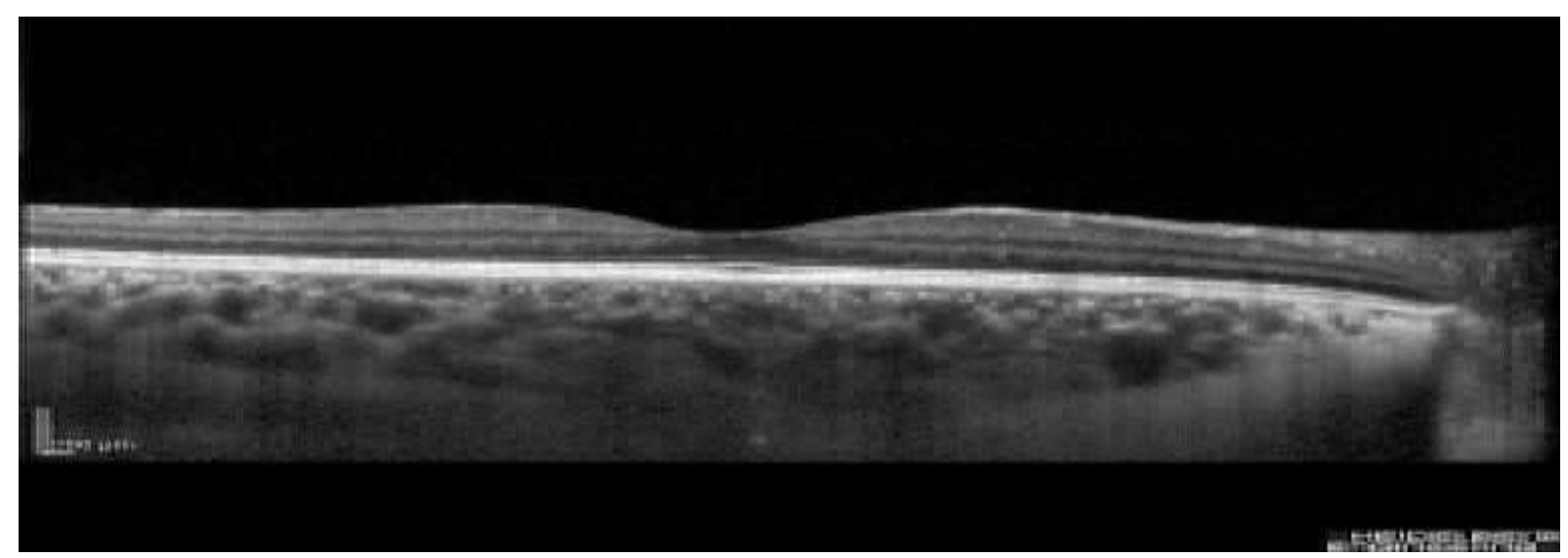

Fig.8:- Denoised from our algorithm

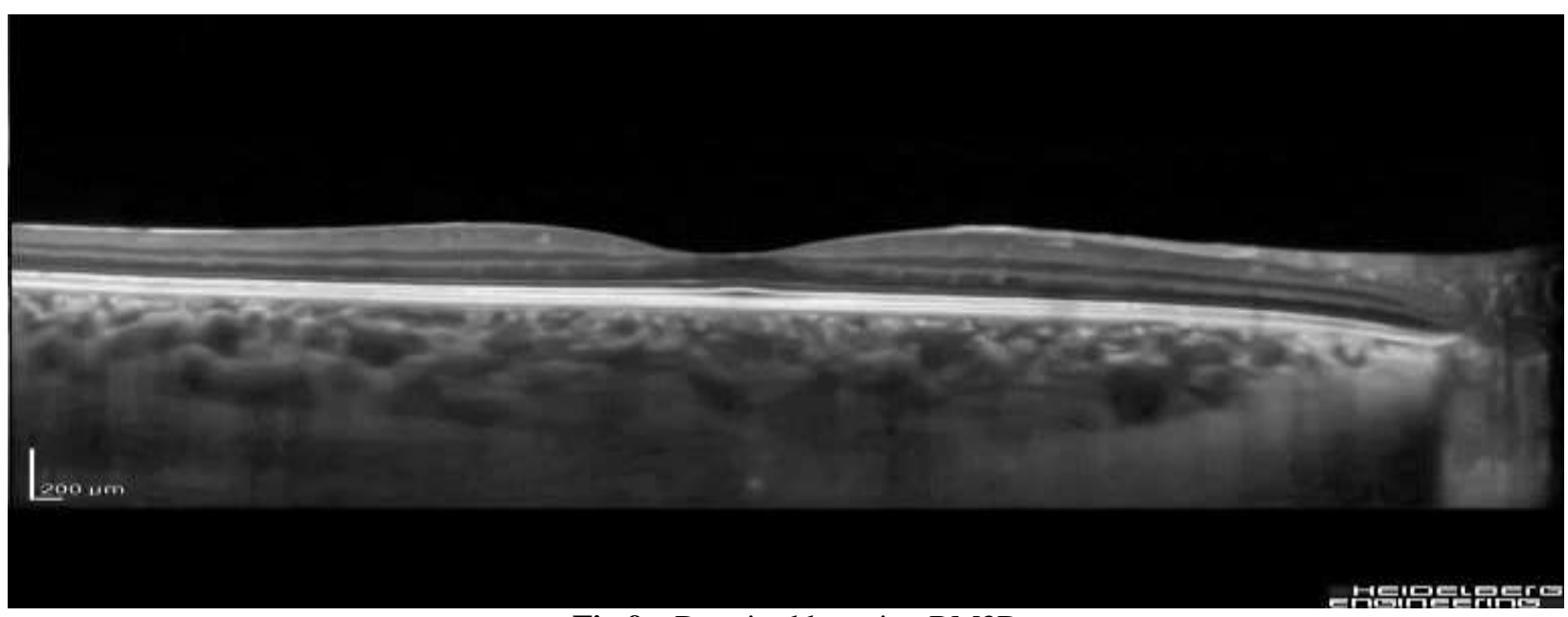

Fig.9:- Denoised by using BM3D

\section{Conclusion:-}

The assumptions that the noise distribution is Gaussian, and the darker regions contain more noise than Information is working well with three level decomposition are working well to produce reasonable approximate images with good edge information.

\section{References:-}

1. H. Rabbani and M. Vafadust, "Image/video denoising based on a mixture of Laplace distributions with local parameters in multidimensional complex wavelet domain," Signal Processing, vol. 88, no. 1, pp. 158-173,2008.

2. N. R. Mahajan, R. C. R. Donapati, S. S. Channappayya, S. Vanjari, A. Richhariya, and J. Chhablani, "An automated algorithm for blood vessel count and area measurement in 2-d choroidal" scan images in Engineering in Medicine and Biology Society (EMBC), 2013 35th Annual International Conference of the IEEE. IEEE, 2013, pp. 3355-3358

3. N. Otsu, "A threshold selection method from gray-level histograms" Automatica, vol. 11, no. 285-296, pp. 2327, 1975.

4. D. L. Donoho, "De-noising by soft-thresholding" Information Theory IEEE Transactions on, vol. 41, no. 3, pp. 613-627, 1995

5. L. Haar, NBS/NRC steam tables. CRC Press, 1984.

6. I. Daubechies et al., Ten lectures on wavelets. SIAM, 1992, vol. 61. 